Improvisation facilitates divergent thinking and creativity: Realising a benefit of primary school arts education

Paul T. Sowden ${ }^{1}$, Lucie Clements ${ }^{2}$, Chriselle Redlich ${ }^{3}$ and Carine Lewis ${ }^{3}$

1. School of Psychology, University of Surrey, Guildford, Surrey, GU2 7XH, UK

2. Trinity Laban Conservatoire of Music and Dance, Faculty of Dance, Laban Building, Creekside, London SE8 3DZ, UK

3. Department of Psychology, University of Hertfordshire, Hatfield, Hertfordshire, AL10 9AB, UK

In press: Psychology of Aesthetics, Creativity and the Arts (http://www.apa.org/pubs/journals/aca/)

\title{
Copyright APA
}

This article may not exactly replicate the final version published in the APA journal. It is not the copy of record.

Correspondence concerning this article should be addressed to Paul Sowden, Department of Psychology, University of Surrey, Guildford, Surrey, GU2 7XH, UK. Email: p.sowden@surrey.ac.uk 
Running head: Improvisation, divergent thinking, creativity and education

\begin{abstract}
The benefit of arts education for cultural engagement, wider academic achievement, and as a contributor to the creative economy is a subject of significant debate. In the present work we focus on the potential for simple arts-based improvisation activities to enhance divergent thinking skills and creativity in primary school age children. In a first experiment we compared the effect of children taking part in an improvised vs. non-improvised dance class on their subsequent performance on the Instances Task (Wallach \& Kogan, 1965) and on a creative 'toy' design task. In a second experiment children took part in verbal and acting improvisation games or in matched control games before completing figural activity 1 of the Torrance Tests of Creative Thinking (Torrance, 1974). In both experiments we found that children who took part in the improvisation interventions showed better divergent thinking and creativity after the intervention. Our findings suggest that simple, arts-based improvisation interventions could have domain general benefits for creative cognition processes. Further, they indicate one way in which simply making better use of existing arts education provision could provide a cost-effective way to increase creativity relevant skills in primary school children. We consider putative mechanisms for the improvisation effects and specify directions for future work.
\end{abstract}

Key Words: Improvisation, Creativity, Divergent Thinking, Arts, Education, Culture 
Running head: Improvisation, divergent thinking, creativity and education

Improvisation facilitates divergent thinking and creativity: Realising a benefit of primary school arts education

\section{Introduction}

The place of creativity in mainstream education and, within that, the role of arts education has been a focus of debate in recent years (President's Committee on the Arts and Humanities, 2011; Henley, 2012; Warwick Commission, 2015). On the one hand, there is accumulating evidence for the wide benefits of arts education (Asbury \& Rich, 2008; Catterall, Dumais, \& Hampden-Thompson, 2012; Winner \& Vincent-Lancrin, 2013; Vaughn \& Winner, 2000) and on the other a 'patchy' and reducing pattern of provision (Warwick Commission, 2015). The National Endowment for the Arts (NEA) note that "keeping the arts as central to the curriculum is an ongoing challenge. A "wicked problem," to be sure" (NEA, 2014, p.3). In the UK, the most recent version of the National Curriculum (Department for Education, 2013a) does not require children to take formal qualifications in arts subjects and seems to place increasing emphasis on acquiring knowledge through 'traditional' approaches rather than on learning to think. An implicit assumption appears to be that knowledge should come before creativity. To many, this view might seem common sense and it reflects the long held idea that to achieve eminent expertise requires a very significant investment of time, around 10,000 hours or 10 years (Ericsson, Krampe \& Tesch-Römer, 1993; Simon \& Chase, 1973). However, recent work has indicated that domain general cognitive abilities, such as working memory capacity, may also be a pre-requisite to achieve expertise (Hambrick, Oswald, Altmann, Meinz, Gobet \& Campitelli, 2014). Thus, it seems that, domain specific practice alone is not sufficient to explain, or guarantee, expertise and creative success.

In contrast to the reducing opportunities for learning to think creatively in education, it has been noted that fostering creativity in education is essential for economic growth and the social good (Craft, 2003; NESTA, 2012; Roberts, 2006; Robinson, 1999; Partnership for 21st Century Skills, 2008). Part of this benefit may be realised through the capacity of creative activities to help develop 
Running head: Improvisation, divergent thinking, creativity and education

children's cognitive abilities (Boyes \& Reid, 2005; Catterrall, Chaplow \& Iwanaga, 1999; Dunbar, 2004; Hassler, 1992; Keinanen, Hetland \& Winner, 2000; Schellenberg, 2001; Smithrim \& Upitis, 2005; Vaughn, 2000) as well as their creative achievement (Minton, 2002). However, it remains a matter of debate to what extent the beneficial effects of creative arts activities on cognitive skills are domain (music, drama, visual arts etc; e.g. Koutsoupidou and Hargreaves, 2009) and modality specific (e.g. spatial, verbal, visual etc.; Jaschke, Eggermont, Honing \& Scherder, 2013; Jonides, 2008; Minton, 2002; Lewis \& Lovatt, 2013; Winner \& Cooper, 2000) with a call for stronger experimental studies in the area. Overall, despite the emphasis of current educational policy on acquiring knowledge, there is substantial evidence that there are potential gains to be made from creative arts and cultural activities in education and there is a need to further evaluate the extent to which they promote domain general cognitive skills such as those that underpin creative thinking ability.

Creativity has been defined as "the interaction among aptitude, process and environment by which an individual or group produces a perceptible product that is both novel and useful as defined within a social context" (Plucker, Beghetto \& Dow, 2004, p.90). At the core of this definition are the dual criteria that creative outcomes are at least novel and useful or relevant (Runco \& Jaeger, 2012). Similarly, most theories of the creative thinking process have at their core dual processes to facilitate generation of novel ideas and to evaluate them in order to produce ideas that will be of value to the context in which they are generated (e.g. Campbell, 1960; Finke, Ward \& Smith, 1992; Gabora, 2005; Guilford, 1956; Howard-Jones, 2002; Simonton, 1999; see Sowden, Pringle \& Gabora, 2015 for a synthesis). Thus, when considering interventions to foster creativity, it is important to specify which aspects of the creative process those interventions aim to improve. In the present experiments we look at the potential for creative arts based improvisation activities in primary education to facilitate divergent thinking processes specifically as well as to enhance the overall creative output. 
Running head: Improvisation, divergent thinking, creativity and education

Our focus on divergent thinking arises because evidence indicates that divergent thinking is a key component of the creative thinking process that predicts creative achievement (Jauk, Benedek, Neubauer, 2014; Plucker, 1999). There has been a long history of work to explore ways of improving divergent thinking ability in both adults and children. In a meta-analysis, Scott, Leritz and Mumford (2004) identify positive effects of creativity training programmes on divergent thinking (e.g. fluency and originality of ideas). They argue that creativity training programmes should be based on learning about specific heuristics or strategies for working with pre-existing knowledge coupled with illustrations of how these work in real-world domains and practice at applying them to the domain at hand. This reflects a domain specific view of creativity (e.g. Baer, 1998). In support of this view, in Scott et al's analysis, more open exploratory techniques, where less concrete information about their application was given, were either unrelated or negatively related to outcomes on particular aspects of the creative process.

Nevertheless, a variety of work, published since the analysis of Scott et al. (2014) suggests that a range of variables may exert an indirect influence on divergent thinking and creative outcomes (e.g. Ritter, Damian, Simonton, van Baaren, Strick, Derks, \& Dijksterhuis, 2012; Steidle and Werth, 2013; Vohs, Redden, \& Rahinal, 2013). Of particular note, is the extensive literature showing a relationship between positive activating moods and divergent thinking (Baas, De Dreu \& Nijstad, 2008; Davis, 2009). These findings would appear to support more domain general influences on creative thinking. In a similar vein, in the present work we explore the possibility of domain general improvements following improvisation activities.

Improvisation can be defined as the process of creating ideas spontaneously - on the spot - without the allowance of planning or refinement of the creative idea. The emerging product is seen as the original thought process that occurred and as such is regarded to be the raw creative process (Eisenberg \& Thompson, 2003; Nooshin, 2003). Our focus on improvisation arises because work in the domain of musical improvisation has shown that improvisation and divergent thinking ability are 
Running head: Improvisation, divergent thinking, creativity and education

strongly related. For instance, in a review of neuroimaging evidence on the neural correlates of improvisation, Beatty (2015) notes that one of the most consistently activated areas is the Inferior Frontal Gyrus, a brain area that is also strongly associated with divergent thinking in creative idea generation tasks (Gonen-Yaacovi, de Souza, Levy, Urbanski, Josse, \& Volle, 2013). Further, Beaty, Smeekens, Silvia, Hodges and Kane (2013) found a strong relationship between divergent thinking ability and musical improvisation, such that divergent thinking ability predicted $57 \%$ of the variance in expert ratings of the creative quality of improvisation. Here we examine the possibility of the converse relationship, by investigating whether improvisation promotes divergent thinking.

Finally, we have chosen to focus on primary education (4-11 years) for two main reasons. First, primary education represents a formative period in a child's development and one during which many are open to the opportunities and experiences afforded by creative arts activities. Second, whilst there is a considerable degree of specification in the primary curriculum and an emphasis on knowledge acquisition in many areas, there is still compulsory integration of creative arts activities, such as music, throughout this stage of education and these types of activities afford the opportunity to more readily integrate improvisation based approaches within existing practise.

\section{Experiment 1: The effect of dance improvisation on divergent thinking and creativity}

In our first experiment we were interested to explore the impact of participating in improvisation dance activities on divergent thinking and creative outcomes. At primary school level in the UK, dance is taught as part of the physical education curriculum (Department for Education, 2013b), which states that children should be able to "perform dances using simple..." (Key Stage 1; 4-7 years; p.2) or "a range of movement patterns" (Key Stage 2; 8-11 years; p.2). Dance is often taught on the National Curriculum in a 'command' style, where the teacher makes decisions and the pupils follow these decisions on cue (Mosston \& Ashworth, 2002). The emphasis is typically on fitness and skill. Yet dance is an aesthetic experience and a creative process, through which the body, brain and personality combine to express and communicate thoughts and feelings ( $H^{\prime}$ Doubler, 1966). Dance 
Running head: Improvisation, divergent thinking, creativity and education

relies on cognitive processes such as communication of abstract concepts, emotional expression and creation of symbols and cannot occur without thinking, feeling and knowing (Hanna, 1983). Thus, Sharp and Le Metais $(2000, p .7)$ note that "dance is an expressive subject, which does not fit well within a subject area focused on physical exercise and sport.... to the exclusion of the creative and expressive nature of dance".

Consequently, we were interested to explore whether dance taught in a more flexible manner, befitting its inherent creative potential, might have benefits beyond the improvements in fitness, health and skill that are emphasised by physical education. Interestingly, there are hints in previous work that support this possibility. For instance, Minton (2002) found that High School students who participated in dance classes for one semester (5-8 hours per week) showed more improved scores on the Torrance Tests of Creative Thinking (TTCT, Torrance, 1974) figural forms than students who did not study dance. However, the work did not distinguish improvisation from technique focused classes. In addition, Kim (2002) compared seventh-grade girls taking traditional and creative dance classes (45 minute classes, twice a week for eight weeks). Girls in the creative dance classes showed significantly greater improvement on the TTCT figural forms. Thus, these studies provide some evidence for a benefit of dance on divergent thinking but it is unclear to what extent improvisation in dance is particularly beneficial. Further, whilst these studies explored the impact of extended dance classes we were interested in whether more immediate effects might be possible.

Consequently, we were interested to test whether improvisation based dance activities in particular could have benefits for aspects of the creative thinking process and, in particular, for divergent thinking. Further, we were interested to see if these benefits could be realised after just one session of improvisation, as suggested by the co-activation of specific brain areas by both divergent thinking and (musical) improvisation (Beaty, 2015). Finally, we were interested to know whether any benefits of improvisation transferred to divergent thinking in another modality. Dance can be considered a spatial activity and so the improvement in performance on figural forms of the TTCT found in 
Running head: Improvisation, divergent thinking, creativity and education

previous work could conceivably reflect an improvement in spatial processing as a result of participating in dance activities. We were interested to see if any benefits of improvisation dance activities generalised beyond the spatial domain. Consequently, in the present study we measured divergent thinking and creativity using both a verbal and a spatial task.

Overall, our experiment will begin to address the issues we raise in our introduction concerning whether improvisation can benefit divergent thinking, whether these benefits can be extended beyond the specific improvisation domain and therefore whether this might support the role of arts based improvisation activities with primary school education.

\section{Method}

Participants

The sample consisted of 27 children enrolled full time in one of two mainstream primary schools. None were learning dance apart from taking part in dance activities as part of the Key Stage 2 (7-11 years) physical education curriculum. Permission was sought from the head teacher of each school and parental consent obtained for each child to participate. There were 17 male and 10 female participants and their ages ranged from $8-10$ years $(M=9.00, S D=.62)$.

\section{Materials}

Personality Assessment: Previous research has shown that personality traits explain some of the variance in divergent thinking performance and creativity (Feist, 1998; Furnham, Crump, Batey \& Chamorro-Premuzic, 2009). Consequently, the Adjusted NEO- Five- Factor Inventory (Adjusted NEOFFI; Markey et al., 2002) was used to assess personality. The 60 item Adjusted NEO-FFI has been developed to measure childrens' personality and correlates with the NEO- Five- Factor Inventory (NEO-FFI; McCrae \& Costa, 1989). Both the NEO-FFI and Adjusted NEO- FFI measure the five basic personality factors (openness to experience, conscientiousness, extraversion, agreeableness and neuroticism) with 12 items per trait. The Adjusted NEO-FFI is self-reported using a five point likert 
Running head: Improvisation, divergent thinking, creativity and education

scale. The present sample was a little younger than Markey et al. so all participants were given the opportunity to ask questions regarding any language they did not understand.

Intelligence Assessment: Intelligence has been shown to predict some of the variance in divergent thinking scores (Kim, 2005; Silvia, 2008). Consequently, Raven's Coloured Progressive Matrices (Raven, Raven \& Court, 1962) were used to obtain a measure of general analytic intelligence.

Mood Assessment: We assessed participants' mood using the Self-Assessment Manikin (SAM; Bradley \& Lang, 1994). The SAM is a nine-point non-verbal, pictorial measure of pleasure, arousal and dominance. Here we were interested in scores on the pleasure and arousal dimensions as previous work suggests that positive, activating moods may benefit divergent thinking (Baas et al., 2008).

Measurement of divergent thinking and creativity: The Instances Task (Wallach \& Kogan, 1965) was used to measure childrens' divergent thinking ability. This asks them to write down, in turn, all the things they can think of that are round, make a noise, that are square, and that move on wheels. No time limit was given for the task, but all participants reached a natural end within ten minutes. To measure overall creativity, we adapted the Product Design Task reported in Mehta and Zhu (2009). Participants were provided with a set of 20 shapes. From these, five were selected by the participant to create a 'new and exciting toy'. Participants were asked to give their toy design a title "so it is different to everyone else's".

Design

At each school, boys and girls were separately randomly allocated to either a control or improvisation dance condition. This ensured approximately equal numbers of boys and girls across the two conditions ( 8 boys and 5 girls in the control condition; 9 boys and 5 girls in the improvisation condition).

Procedure 
Running head: Improvisation, divergent thinking, creativity and education

Prior to the testing session, all childrens' parents were sent an information sheet to fully explain the details of the study and a consent form for them to sign if they wished their child to participate. Prior to taking part all children were given simple written instructions, which were also read out. After an opportunity to ask questions and to have any aspects of the research that they did not understand explained to them, the children were asked to tick a sheet with their name on in order to confirm their willingness to take part. Only one child did not wish to participate. In total, testing lasted approximately 60 minutes. Half the children completed the Raven's Coloured Matrices and the Adjusted NEO-FFI before taking part in the dance condition and completing the measures of mood and creativity. The other half of the children completed the Raven's and Adjusted NEO-FFI after dancing and all other measures, such that the order of completion was fully counterbalanced with dance condition across the two schools. Children completed the Raven's and Adjusted NEO-FFI in their classroom seated separately with space in between them. All other measures were completed in the school hall. Participants completed the dance task in groups of 6 or 7 . The remainder of the tasks were completed on an individual basis, although the group of participants remained in the same room.

Upon entering the school hall, participants were asked to participate in a ten minute dance task. In order to hold constant the teacher for both groups of children, and due to the unusual nature of the improvisation task which differed from the usual teaching style of the national physical education curriculum, an experienced dancer led the dance task. In the control dance condition children took part in a 10 minute command style dance task. This involved copying the dancer in a number of different movements, which resulted in learning a simple routine. This was based upon a task previously used with adults (Lewis, 2012; p.116), although movements thought inappropriate for children were excluded. Participants danced to three recent pop songs. In the improvisation dance condition participants completed a series of dance improvisation exercises, for 10 minutes, each of which asked the participants to use a specified part of their body to explore as many spontaneous ways of moving as possible that differed from their usual way of moving. The method again followed 
Running head: Improvisation, divergent thinking, creativity and education

that of Lewis (2012; p.116). Children danced to the same three pop songs as in the control condition. After dancing the children remained in the hall and were asked to complete the SAM, the Instances Task, and the adapted Product Design Task. Children were seated with space in between them and their neighbour. Upon completion of all tasks, participants were debriefed and offered stickers to thank them for their participation. They were not informed of the stickers prior to participating in order to discourage extrinsically motivated participation.

\section{Scoring Procedure}

Fluency of responding for each item on the Instances Task was measured by counting the total number of valid answers provided to each question. To assess originality for each item on the Instances Task, the number of times a given response occurred across the entire sample was divided by the sample size and the result subtracted from one (Zenasni \& Lubart, 2009). Thus, scores tending towards 1.0 indicate more unique ideas and, conversely, a score of zero indicates an idea that the entire sample had. Calculating the mean originality across all the responses produced by a participant for each item on the Instances Task provided their mean originality score for that item. Averaging in this way avoids the problems of scoring methods that sum originality scores across a participant's responses thereby confounding fluency and originality (see Silvia et al., 2008). Finally, overall creativity of responses on the Instances Task was measured through the use of the Consensual Assessment Technique (CAT; Amabile, 1982). The CAT was also used to measure the creativity and originality of the Toy Design Task. The CAT was carried out by two male and three female raters who were psychology students at the University of Surrey. To evaluate the level of creativity exhibited on the Instances Task, each rater was asked to score the set of answers provided to each of the four items. The raters were asked to score between 1 (low) and 6 (high), how creative they believed the each answer to be. The mean of the five raters' scores was calculated for each answer and participant. Scores were then averaged across answers, to provide a score for each of 
Running head: Improvisation, divergent thinking, creativity and education

the four items on the Instances Task. The same procedure was used for the Toy Design Task with the addition of a separate CAT rating of originality.

Inter-rater agreement for the CAT ratings was checked using the intra-class correlation coefficient. Agreement for the different measures was high ranging from $\operatorname{ICC}(2,5)=.837,95 \% \mathrm{Cl}[.701, .919]$ to $\operatorname{ICC}(2,5)=.873,95 \% \mathrm{Cl}[.779, .935]$.

\section{Results}

Dependent variables were checked for normality as appropriate and transformations applied when skewness or kurtosis divided by the relevant SE exceeded 1.96. Any such transformations and the resultant skewness and kurtosis values are reported below.

\section{Instances Task}

Previous research has frequently found that fluency and originality are correlated such that the likelihood of producing original responses increases with the total number of responses made (Plucker, Qian \& Wang, 2011). Although our originality scoring procedure should prevent this problem, we nevertheless checked the correlation between fluency and originality for each item on the instances task. In all cases these correlations were small and mostly non-significant (round, $r=.09, p>.05$; noise, $r=.07, p>.05$; square, $r=.33, p>.05$; wheels, $r=.39, p=.04$ ) with the largest, for wheels, showing that the two measures shared only $16 \%$ variance in common. Consequently, originality and fluency were treated separately in the following analyses.

Next we checked whether any of the Big-5 personality dimension scores were correlated with mean fluency, originality or CAT creativity scores. All of the correlations were small and none were significant $\left(-.30<r^{\prime} s<.18, p^{\prime} s>0.05\right)$. Consequently, personality scores were not included as covariates in subsequent analyses.

To assess the potential for an impact of mood on our measures of divergent thinking we first conducted a 2-way analysis of variance (SAM dimension (2) - pleasure, arousal; condition (2) - 
Running head: Improvisation, divergent thinking, creativity and education

control, improvisation), with repeated measures on SAM dimension, to assess whether there were differences on the SAM dimensions as a function of completing the two dance conditions. The SAM dimension $x$ condition interaction was not significant, $F(1,25)=.024, p>.05$ indicating that levels of pleasure and arousal did not differ after completing the two dance conditions. After completing the dance activities both groups reported positive activating mood states (Table 1).

\section{<TABLE 1 ABOUT HERE $>$}

Next we explored the correlation between pleasure and arousal scores, and mean fluency, originality and CAT creativity scores. None of the correlations were significant $\left(-.16<r^{\prime} s<.17, p^{\prime} s>.05\right)$. Consequently, pleasure and arousal scores were not included as covariates in subsequent analyses.

Finally, we assessed whether general IQ, measured using the Raven's, was related to fluency, originality or CAT scores. All correlations were small and non-significant $\left(.16<r^{\prime} s<.32, p^{\prime} s>.05\right)$. Consequently, Raven's scores were not included as co-variates in subsequent analyses.

To explore the effect of dance condition, we used univariate analyses of variance, one for fluency scores, one for originality scores and one for CAT scores. First, we computed average fluency, average originality and average CAT scores across the four items of the Instances Task. Inspection of histograms and calculation of skewness and kurtosis statistics showed that average fluency and originality scores were both skewed and kurtotic for the control condition. Consequently, we used square-root and reflected square-root transformations respectively to correct these values to: control condition fluency skewness=.73 (SE=.62) and kurtosis=1.62 (SE=1.19); improvisation condition fluency skewness=.41 (SE=.60) and kurtosis=-.14 (SE=1.15); control condition originality skewness=1.19 ( $\mathrm{SE}=.62$ ) and kurtosis=.56 ( $\mathrm{SE}=1.19)$; improvisation condition originality skewness=$.85(\mathrm{SE}=.60)$ and kurtosis=1.38 (SE=1.15).

For fluency there was no significant effect of condition, $F(1,25)=1.14, p=.30$. However, for originality the improvisation group produced significantly more original instances, $F(1,25)=7.93, p=.009$, partial- 
$e \operatorname{ta}^{2}=.24, M_{\text {control }}=.74(95 \% \mathrm{Cl}[.68, .80]), M_{\text {improvisation }}=.85(95 \% \mathrm{Cl}[.80, .91])$. A control analysis that included fluency as a covariate, showed that the improvisation group still produced significantly more original instances, $F(1,24)=7.56, p=.011$, partial eta ${ }^{2}=.24$, after controlling for fluency. Finally, CAT scores indicated that the improvisation group were rated as producing significantly more creative toys, $F(1,25)=7.83, p=.01$, partial eta ${ }^{2}=.24, M_{\text {control }}=2.45(95 \% \mathrm{Cl}[2.15,2.75])$,

$M_{\text {improvisation }}=3.01(95 \% \mathrm{Cl}[2.72,3.29]$

Toy design task

As for the instances task, we first checked whether any of the Big-5 personality scores, pleasure and arousal scores, and Raven's scores were correlated with the CAT creativity or originality ratings.

There were no significant correlations (creativity, $-.38<r^{\prime} s<.35, p^{\prime} s>.05$; originality, $-.17<r^{\prime} s<.35$, $p$ 's $>.05)$. Consequently, none of these measures were included as covariates in subsequent analyses.

We also looked at the correlation between CAT creativity and CAT originality ratings. As might be expected, there was a significant correlation $(r=.58, p=.001)$. However, given that the two measures only shared $34 \%$ of their variance in common this supports the assumption that the CAT creativity measure is capturing more than just the originality of the participants' responses.

To explore the effect of dance condition on originality and creativity we used univariate analyses of variance. The improvisation group were rated as producing significantly more original, $F(1,25)=15.93, p=.001$, partial-eta ${ }^{2}=.39, M_{\text {control }}=2.28(95 \% \mathrm{Cl}[1.80,2.75]), M_{\text {improvisation }}=3.56(95 \% \mathrm{Cl}$ $[3.10,4.02])$, and creative toy designs, $F(1,25)=9.78, p=.004$, partial-eta ${ }^{2}=.28, M_{\text {control }}=2.75(95 \% \mathrm{Cl}$ $[2.40,3.11]), M_{\text {improvisation }}=3.50(95 \% \mathrm{Cl}[3.16,3.84])$.

\section{Discussion}

Overall, the results of Experiment 1 indicate that taking part in a 10-minute improvisation dance class benefits subsequent originality and creativity on the Instances Task - a measure of divergent 
Running head: Improvisation, divergent thinking, creativity and education

thinking ability - and on a Toy Design Task. Given that previous work has shown divergent thinking is a strong predictor of creativity (Jauk, Benedek, Neubauer, 2014; Plucker, 1999), it is entirely possible that the higher creativity scores were a function of the enhanced divergent thinking of the improvisation group. However, it is also possible that aspects of the creative process beyond divergent thinking were influenced by improvisation, hence benefitting overall creativity. At present this remains an open question. The effects of improvisation in the present study are not explained by between group differences in general IQ or personality as, for the present sample, these measures were not strongly related to creative outcomes. Further, variation in mood does not explain the effects, as both groups reported equivalent, positive activated, mood states after dancing. Whilst previous work has shown a benefit of positive activating mood states for divergent thinking (Baas et al., 2009; Davis, 2009) that was clearly not the whole story in the present case as the benefit of mood would have been shared equally by both groups.

Our findings also demonstrate that the benefits of improvisation for divergent thinking are not specific to the domain or modality of the improvisation task. Neither of the divergent thinking and creativity tasks were in the domain of dance. Further, whilst the Toy Design task was a spatial modality task that might just conceivably have benefitted from the fact that dance is also, in part, a spatial skill, the Instances task required verbal responses and shared neither domain nor modality with the dance improvisation. However, a single result is not sufficient to conclude that the benefits of improvisation for divergent thinking and creativity are domain general. Consequently, we next report a further experiment that explores this issue, this time exploring whether taking part in verbal and acting games benefit a subsequent figural divergent thinking task. In addition, Experiment 2 addresses a weakness in the design of Experiment 1. We did not pre-test the childrens' creativity prior to the dance intervention. Whilst our random assignment of boys and girls to condition should have reduced the possibility of pre-existing between group differences in divergent thinking and creative ability accounting for the observed post-dance differences, it is still a possibility. By using a pre-test, post-test design Experiment 2 controls for this concern. 
Running head: Improvisation, divergent thinking, creativity and education

\section{Experiment 2: The effect of verbal improvisation and acting on divergent thinking}

In the present experiment we explore the impact of verbal improvisation and acting on divergent thinking. Verbal improvisation is the act of spontaneously creating strings of new words, while avoiding pre-planned phrases (Sawyer, 2008). Lewis and Lovatt (2013) suggest that verbal improvisation shares in common with everyday speech the use of general knowledge structures or schemas (Bartlett, 1932). However, when improvising these schemas are reformulated to produce something new (Pressing, 1987). Consequently, rather than a conversation that uses set phrases of speech linked to a well-worn schema, improvisation draws on a wider range of schemas and reformulates these, presenting more choice in speech and making it more creative.

There are a few findings in the literature that suggest verbal improvisation might benefit subsequent divergent thinking. Schmidt, Goforth and Drew (1975) compared two groups of $5 \frac{1}{2}-6$ year old children, who took part in an eight-week creative dramatics course, with an age-matched control group who just completed usual classroom activities. At the end of the course, children taking the creative dramatics course showed more fluent responses on two measures of divergent thinking, both of which required verbal responses. Lewis and Lovatt (2013) also explored the impact of verbal improvisation and drama on divergent thinking, but over a much shorter time frame. They had 41 undergraduate psychology students participate for 20 minutes in verbal improvisation or matched verbal games that did not require improvisation and found that the improvisation group showed more improvement on an Alternate Uses Task (Guilford, Christensen, Merrifield, \& Wilson, 1971). Like Schmidt et al both the improvisation and divergent thinking task were verbal. Karakelle (2009) took a group of 30 postgraduate students, of whom half completed a 10-week drama course. The students completing the drama course showed significantly increased divergent thinking scores on an alternate uses and figural drawing task one week after completing the drama course. Thus, unlike Schmidt et al., and Lewis and Lovatt, Karakelle showed that the positive benefit of the drama 
improvisation extended to a different response modality; from verbal to figural. However, as the students self-selected the drama course it is possible that other factors, such as personality traits, could explain the positive findings.

Thus, similar to Experiment 1 in the present paper, and to Lewis and Lovatt (2013), we were interested to test once more whether short periods of improvisation are sufficient to benefit divergent thinking and creativity but using different improvisation tasks and measures of divergent thinking. This will test whether the effects observed in Experiment 1 generalise. Further, we wished to see whether these short time-frame effects would transfer across domain and modality in the present primary school age population.

\section{Method}

Participants

The sample consisted of 34 children enrolled full time in a single mainstream primary school. Participants were recruited by seeking permission from the head teacher of each school and then gaining parental consent for each child. All participants were in one of two Year 6 (age 10-11 years) classes.

\section{Design and materials}

Participants were randomly allocated to either a control or an improvisation condition. They completed a different version of the Incomplete Figures Task, counterbalanced across participants, before and after taking part in verbal and acting activities tailored to their condition. The Incomplete Figures Task, taken from the Torrance Tests of Creative Thinking, was used to assess non-verbal divergent thinking ability. Each form presents a piece of paper with an incomplete shape on. The child was asked to finish off the picture however they pleased within three minutes and give their picture a title. 
Running head: Improvisation, divergent thinking, creativity and education

\section{Procedure}

Prior to the testing session, all childrens' parents were sent an information sheet to fully explain the details of the study and a consent form for them to sign if they wished their child to participate. At the start of the testing session all children were given simple instructions about the tasks that they would perform. They were given an opportunity to ask questions and to have any aspects of the research that they did not understand explained to them. Before participating, all children were reminded that they could stop taking part at any time and were told that it was not a test but just fun tasks. All children then completed one version of the Incomplete Figures Task. Following this, the children were randomly divided into two groups, an improvisation group and a non-improvisation group. Each group played a series of verbal and acting games, "No, you can't take me!", the 'Social Roles Game' and 'Whole Zoo Duck Duck Goose'. These were adapted to create improvisation and non-improvisation versions of each game.

The improvisation versions of each game required participants to act parts and/or verbally improvise. For instance, in "No, you can't take me!" each participant secretly chose one object that would be found in a given room (e.g. if they were given 'kitchen', one child might choose 'sink'). When the game leader then exclaimed " $M y$, look at all this useless stuff! I've got to get rid of some of this junk!" the participant had to reply "NO, YOU CAN'T TAKE ME!" and then, without mentioning the name of the object, improvise a reason why not. e.g. "If you take me away, no one can wash their hands". The improvised exchange would then continue for a while and other participants tried to guess the secret object. Non improvised versions of games simply required the children to do things such as factually describe objects in their room or social roles that they were given, whilst the other children tried to guess what was being described.

After playing the improvisation games the children were administered the other version of the Incomplete Figures Task that they did not do before. Finally, the children were thanked, verbally debriefed, told that they did very well and they were given a sticker. 
Running head: Improvisation, divergent thinking, creativity and education

\section{Scoring procedure}

The Incomplete Figures Tasks were scored according to the Streamlined Scoring Manual for the Figural TTCT to provide scores for originality, elaboration and abstractness of titles.

Inter-rater agreement for elaboration and abstractness of titles was checked using the intra-class correlation coefficient. Agreement was high for elaboration $\operatorname{ICC}(2,67)=.83,95 \% \mathrm{Cl}[.64, .92]$ and abstractness of titles $\operatorname{ICC}(2,67)=.78,95 \% \mathrm{Cl}[.57, .89]$.

\section{Results}

Although participants were randomly assigned to conditions it is still possible that there were preintervention differences in their performance on the divergent thinking measures. Consequently, we conducted three between groups t-tests, one for each of the three figural TTCT scores. There were no significant differences between conditions on originality or abstractness of titles. However, the improvisation condition had significantly higher baseline elaboration scores, $t(32)=2.66, p=.012$, $M_{\text {control }}=1.67(95 \% \mathrm{Cl}[.89,2.44]), M_{\text {improvisation }}=3.19(95 \% \mathrm{Cl}[2.38,3.99])$. Work to compare methods of analysing designs with random allocation to conditions and both pre and post intervention scores, shows that analysis of covariance (ANCOVA) estimates are unbiased, more powerful and more precise than repeated-measures ANOVA or change score analysis, especially when there is an initial baseline imbalance (Egbewale, Lewis \& Sim, 2014; van Breukelen, 2006). Consequently, we used ANCOVA's here, one for each of the three figural TTCT scores, to control for baseline differences in pre-intervention scores. In each case the relevant pre-games figural TTCT score was entered as a covariate in the analysis. Participants in the improvisation condition showed significantly higher post-games originality scores after controlling for pre-games originality, $F(1,31)=7.09, p=.012$, $\eta_{p}{ }^{2}=.19, M_{\text {control }}=.86(95 \% \mathrm{Cl}[.45,1.27]), M_{\text {improvisation }}=1.66$ (95\% Cl $\left.[1.22,2.09]\right)$. Participants in the improvisation condition showed significantly higher post-games elaboration scores after controlling for pre-games elaboration, $F(1,31)=6.24, p=.018, \eta_{p}{ }^{2}=.17, M_{\text {control }}=2.22(95 \% \mathrm{Cl}[1.40,3.04])$, 
Running head: Improvisation, divergent thinking, creativity and education

$M_{\text {improvisation }}=3.76(95 \% \mathrm{Cl}[2.88,4.63])$. There was no significant difference between conditions in post-games scores for abstractness of titles after controlling for pre-games scores.

\section{Discussion}

Overall, experiment 2 found that following a series of verbal improvisation games, children scored higher on a figural divergent thinking task in terms of originality and elaboration. Further, unlike experiment 1 , this study controlled for pre-intervention divergent thinking scores. These findings generalise the findings of Experiment 1 by suggesting that taking part in a different type of brief improvisation activities can similarly result in an improvement in divergent thinking scores for a different domain of cognition, in this case transferring from verbal and acting improvisation to figural divergent thinking. Thus, these findings provide further support to those of Karakelle (2009) but importantly, show that effects can occur in children and within a short time frame.

However, it is important to be cautious with the present findings because, although the present experiment did control for pre-intervention divergent thinking scores, it didn't control for possible pre-intervention differences in general intelligence or personality. Therefore, despite the random allocation to conditions, there could still have been unmeasured residual differences in these scores that contributed to the between condition difference in baseline elaboration scores. Follow-up work is clearly required. Nevertheless, experiment one did control for these factors and, further, the groups did not differ in the present experiment on their baseline originality or abstractness of titles scores. These observations suggest that general intelligence and personality were not necessarily influencing factors in the present context.

\section{General Discussion}

Our findings have two main sets of implications. First and foremost the present experiments show that taking part in improvisation activities has general benefits for subsequent divergent thinking and creativity in children that transcend the improvisation domain. These benefits can occur after a 
Running head: Improvisation, divergent thinking, creativity and education

remarkably short improvisation intervention. Second, our findings contribute to the wider debate about the value of the arts in education by serving to emphasise the potential inter-relationship between these activities and other creative thinking skills with the ensuing potential for benefits across wider areas of the curriculum. In the following sections we consider these implications more fully.

\section{Benefits and mechanisms of improvisation for divergent thinking and creativity}

The present findings suggest a domain general aspect of creative cognition with respect to childrens' divergent thinking ability and creativity. In their Amusement Park Theoretical (APT) model of creativity, Baer and Kaufman (2005) argue that to be creative in any domain requires certain core attributes such as sufficient intelligence and motivation. At this level the ability to be creative is domain general. However, they argue that as we move towards considering creative achievement in specific domains, and highly specific micro-domains, then a more specific set of attributes become predictive of success in that domain and these attributes do not necessarily predict success in other micro-domains. For instance, expertise about the history of English country house landscape gardens of the 1700's may not help a garden designer working with modern suburban backyards. Thus, a prediction of the APT model would be that with increasing specialisation, comes a requirement for increasingly specific methods of training creativity. Conversely, with little specialisation more general methods of creativity training might be effective. Convergently, in their Four C model of creativity, Kaufman and Beghetto (2009) distinguish mini-c creativity (personal creative insights), little-c (creativity that someone else would recognise like a new family meal), Pro-c (creativity an expert in the domain would recognise as a new and valuable contribution) and Big-C creativity (eminent achievement that few can equal). They note that mini-c is likely to be experienced early in life and that in common with little-c may show both domain specificity and generality, whereas Pro-c and Big- $\mathrm{C}$ are likely to be domain specific due to the increasing practise and expertise required to achieve these levels of creativity. 
Running head: Improvisation, divergent thinking, creativity and education

The divergent thinking and creativity tests used in the present experiments appear firmly rooted at the level of little-c. Further, in primary school most creative achievements are likely to be at the mini and little-c levels. Whilst children have already begun the process of acquiring domain specific knowledge and skills, there is reason to believe that their domain general creative ability is likely to support creative activity across a number of domains, suggesting that more domain general measures of creativity are relevant with this age-group. Thus, the present observation that improvisation in one domain transfers to assessment of divergent thinking and creativity in other cognitive domains is consistent with the notion of domain general little-c creative abilities in primary school age children.

However, Plucker (1998) argues that evidence for domain specificity vs. generality may partially reflect the method of measurement used such that 'performance-based' measures yield evidence of specificity and more traditional assessments (e.g. domain general divergent thinking measures), yield evidence of generality. Put simply, what experimenters find may be a function of what they measure. Clearly, it is possible that, in the present experiments, our inference that little-c creativity can be domain general is likely to be somewhat determined by our choice of relatively general measures of divergent thinking and creativity. Thus, future work now needs to contrast domain specific and domain general measures within a single experiment. For instance, if we had used a dance divergent thinking task following dance improvisation (vs. control) we might have observed an even more pronounced benefit of improvisation on such a domain specific measure compared to the benefit on more domain general measures.

The present findings also suggest that even a short exposure to improvisation activities can help divergent thinking and creativity. This might seem surprising given that previous work has often focused on lengthier training programmes (e.g. Karakelle, 2009; Kim, 2002; Schmidt, Goforth \& Drew, 1975; van de Kamp, Admiraal, van Drie, \& Rijlaarsdam 2015) and given the assertions of Scott et al. (2004), following their meta-analysis of the training and divergent thinking literature, that 
Running head: Improvisation, divergent thinking, creativity and education

training should be lengthy. Against this is the more recent meta-analysis of creativity training packages by Ma (2006), who finds no effect of duration of training. One potential reconciliation is that creativity training packages often teach participants a range of 'tools' that can be applied in the moment to support divergent thinking and creativity. Once a tool has been mastered its effect might be immediate when applied. Improvisation could be seen as one such tool. Similarly, a variety of other factors have been shown to influence creativity in the moment including mood, lighting and office environment (e.g. Baas, 2008; Davis, 2009; Steidle \& Werth, 2013; Vohs et al, 2013). An important question for future work should be to determine the duration of benefit of application of these tools for subsequent divergent thinking and creativity. In the present work the effect was measured immediately after taking part in improvisation activities so it is not clear whether benefits would still be applicable after some intervening period of time.

A further consideration is that the mechanism by which particular tools and influences act on creative thinking are likely to differ as a function of the tool and the stage(s) of the creative process being influenced. For instance, Nijstad, De Dreu, Rietzschel and Baas (2010) propose two routes by which mood could influence creative thinking. Positive, activating moods are proposed to act via a flexibility pathway that increases the range of ideas generated when thinking divergently (see also 'Cognitive Tuning Theory'; Schwarz \& Clore, 2003). Negative activating moods are proposed to act via a persistence pathway that increases the number of a particular type of idea generated when thinking divergently. To this, Sowden \& Dawson (2011) add an additional route by which negative activating moods might influence the evaluation of the usefulness of creative ideas. In the case of improvisation, the mechanism may be rooted in common brain mechanisms underpinning improvisation and divergent thinking. Beaty (2015) identifies that the Inferior Frontal Gyrus (IFG) is an area that is frequently activated in both studies of musical improvisation and in studies of divergent thinking. This may be because the Inferior Frontal Gyrus plays a key role in the retrieval and selection of information from memory (Badre, Poldrack, Paré-Blagoev, Insler \& Wagner, 2005), which would support both improvisation and divergent thinking. Previous work has established that 
Running head: Improvisation, divergent thinking, creativity and education

in the case of priming effects, pre-activation of brain areas can influence subsequent stimulus processing in the pre-activated areas (e.g. Driver \& Frith, 2000). However, there remain several untested assumptions in such an account: (1) that other types of improvisation besides musical improvisation similarly activate the IFG; (2) that pre-activation of IFG during improvisation then endures in some form long enough for the subsequent divergent thinking tasks; (3) that preactivation relating to retrieval and selection of information for one task transfers to a different type of task. To our knowledge these assumptions remain untested in the literature with the possible exception of (1). Liu et al. (2012), in a study of lyrical improvisation by free style rap artists, find associated IFG activation. The verbal character of this improvisation suggests that the role of the IFG in improvisation may extend beyond instrumental improvisation.

At a more cognitive level of explanation the mechanism at work here may be related to schema theory. We all use schemas (Bartlett, 1932) to form our knowledge structures and rely on this process on a day-to-day basis. As such, our thinking process is often structured in a way that promotes a convergent thinking style. Writing on musical improvisation, Pressing (1987) named these schemas, 'referents', described as stored sets of phrases used to help improvisation. The improvisation task therefore, becomes about rearranging these referents in more original ways. In addition, often when people complete a divergent thinking task, the responses become more original as the list goes on (Beaty \& Silvia, 2012). It may be that the act of improvisation facilitates access to more original responses without requiring that people have to go serially through the elements of common schemas associated with the task first of all. Thus, in this case, improvising may have encouraged the children to break away from their schemas or, essentially, their set patterns of thinking, thus leading them to more original responses, straight away, on the subsequent divergent thinking tasks. This break away might happen because the act of improvisation enables people to more readily access unique schemas or, alternatively, as Pressing suggests, schemas may still be used but in a more unusual manner. 
Running head: Improvisation, divergent thinking, creativity and education

Finally, it is worth considering that although we did also find enhanced ratings of creativity in Experiment 1, these may merely reflect the impact of the improvement in divergent thinking ability. Consequently, the extent to which other aspects of the creative process are influenced by improvisation remains an open question that should also be addressed by future work.

\section{Implications for arts education: realising the benefits of existing practice}

The present findings add one further piece of evidence to the picture that arts-based and cultural educational activities should have a central place in the curriculum and be a right of all children, including the many who cannot afford to access these activities outside of school. Previous research has argued that arts-based education can have general benefits for academic attainment (Winner \& Vincent-Lanrin, 2013; Vaughn \& Winner, 2000), as well as benefitting specific non-arts academic subjects (Keinanen, Hetland \& Winner, 2000; Schellenberg, 2001; Smithrim \& Upitis, 2005; Vaughn, 2000). Further, the important role of cultural education in enabling children to develop the creative skills necessary to support the social good and future economic success of the UK has been repeatedly emphasised (Craft, 2003; Henley, 2012; NESTA, 2012; Roberts, 2006; Robinson, 1999; Partnership for $21^{\text {st }}$ Century Skills, 2008; Warwick Commission, 2015.). Despite this emphasis, there has been a steady decline in the provision of arts and cultural education within mainstream education, with increasing reliance on extra-curricular provision that is beyond the reach of lower socio-economic status families (Warwick Commission, 2015).

The present findings suggest one small way in which existing arts-based education activities can be advantageously used to foster more general creative thinking skills. This may be particularly wellsuited to the little-c creativity that is likely to be common at primary school. We suggest that education staff consider adapting the way in which aspects of existing arts based activities are delivered to emphasise improvisation. For instance, by using dance teaching to encourage spontaneity and improvisation, not just fitness and technical skill, significant benefits for divergent thinking skills and creativity could be realised. These types of adaptations are about making better 
Running head: Improvisation, divergent thinking, creativity and education

use of existing provision rather than increasing provision and demonstrate a modest and costeffective way to support creativity relevant skills in primary school children.

\section{Conclusions}

Our findings suggest that a short improvisation intervention is sufficient to enhance performance on subsequent divergent thinking and creativity tasks in children, even when those tasks tap a different cognitive domain. This supports a domain general aspect of creative cognition with respect to childrens' divergent thinking ability and creativity. Hence, the work adds further support to the important role of arts based education activities and emphasises that embedding them fully within the curriculum, so that they are accessible to all, can have benefits that extend beyond the artistic domains themselves. Some benefits, such as those shown in the present work, could be realised simply be making different use of existing provision, thereby demonstrating a cost-effective way to increase creativity skills in primary school children.

\section{References}

Amabile, T. M. (1982). Social psychology of creativity: A consensual assessment technique. Journal of Personality and Social Psychology, 43, 997-1013.

Asbury, C. H., \& Rich, B. (Eds.). (2008). Learning, arts, and the brain: The dana consortium report on arts and cognition. Dana Press.

Baas, M., De Dreu, C.K.W., \& Nijstad, B.A. (2008). A meta-analysis of 25 years of mood-creativity research: Hedonic tone, activation, or regulatory focus? Psychological Bulletin, 134, 779-806.

Badre, D., Poldrack, R.A., Pare-Blagoev, E.J., Insler, R.Z., \& Wagner, A.D., (2005). Dissociable controlled retrieval and generalized selection mechanisms in ventrolateralprefrontal cortex. Neuron, 47, 907-918.

Baer, J. (1998). The case for domain specificity of creativity. Creativity Research Journal, 11, 173-177. Baer, J., \& Kaufman, J. C. (2005). Bridging generality and specificity: The amusement park theoretical (APT) model of creativity. Roeper Review, 27, 158-163. 
Running head: Improvisation, divergent thinking, creativity and education

Bartlett, F. C. (1932). Remembering. Cambridge, UK: Cambridge University Press.

Beaty, R.E. (2015). The neuroscience of musical improvisation. Neuroscience \& Biobehavioral Reviews, 51, 108-117.

Beaty, R. E., \& Silvia, P. J. (2012). Why do ideas get more creative across time? An executive interpretation of the serial order effect in divergent thinking tasks. Psychology of Aesthetics, Creativity, and the Arts, 6(4), 309.

Beaty, R.E., Smeekens, B.A., Silvia, P.J., Hodges, D.A., Kane, M.J., (2013). A first look at the role of domain-general cognitive and creative abilities in jazz improvisation. Psychomusicology: Music, Mind and Brain, 23, 262-268.

Boyes, L. C. \& Reid, I. (2005). What Are the Benefits for Pupils Participating in Arts Activities? The View from the Research Literature. Research in Education, 73, 1-14.

Bradley, M. M., \& Lang, P. J. (1994). Measuring emotion: The self-assessment manikin and the semantic differential. Journal of Behavioural Therapy and Experimental Psychiatry, 25, 49-59.

Campbell, D. T. (1960). Blind variation and selective retention in creative thought as in other knowledge processes. Psychological Review, 67, 380-400.

Catterall, J.S., Dumais, S.A. \& Hampden-Thompson, G. (2012). The Arts and Achievement in At-risk Youth: Findings from Four Longitudinal Studies. Research Report \#55. Washington DC: National Endowment for the Arts.

Catterall, J.S., Chapleau, R., and Iwanaga, J. (1999). Involvement in the Arts and Human Development: General Involvement and Intensive Involvement in Music and Theater Arts. In Deasy, R. (Ed.). Champions of Change: The Impact of the Arts on Learning. Washington, DC: Art Education Partnership.

Craft, A. (2003). The limits to creativity in education: Dilemmas for the educator. British Journal of Educational Studies, 51, 113-127.

Davis, M. A. (2009). Understanding the relationship between mood and creativity: A meta-analysis. Organisational Behavior and Human Decisions Processes, 108, 25-38. 
Running head: Improvisation, divergent thinking, creativity and education

Department for Education, UK (2013a). The National Curriculum in England Framework Document: for Teaching 1 September 2014 to 31 August 2015. London, UK: Department for Education.

Department for Education, UK (2013b). National Curriculum in England; Physical Education Programmes of Study: Key Stages 1 and 2. London, UK: Department for Education.

Driver, J., \& Frith, C. (2000). Shifting baselines in attention research. Nature Reviews Neuroscience, 1, 147-148.

Dunbar, K. N. (2008). Arts education, the brain, and language. In C. Asbury \& C. Rich (Eds.) Learning, Arts, and the Brain. New York, NY: Dana Foundation. pp. 81-92.

Egbewale, B. E., Lewis, M., \& Sim, J. (2014). Bias, precision and statistical power of analysis of covariance in the analysis of randomized trials with baseline imbalance: a simulation study. BMC Medical Research Methodology, 14, 49.

Eisenberg, J., \& Thompson, W. (2003). A Matter of Taste: Evaluating Improvised Music. Creativity Research Journal, 15, 287-296.

Ericsson, K. A., Krampe, R. Th., \& Tesch-Römer, C. (1993). The role of deliberate practice in the acquisition of expert performance. Psychological Review, 100, 363-406.

Feist, G.J. (1998). A meta-analysis of personality in scientific and artistic creativity. Personality and Social Psychology Review, 2, 290-309.

Finke, R. A., Ward, T. B., \& Smith, S. M. (1992). Creative Cognition: Theory, Research and Applications. Cambridge, MA: MIT Press.

Furnham, A., Crump, J., Batey, M., \& Chamorro-Premuzic, T. (2009). Personality and ability predictors of the "consequences" test of divergent thinking in a large non-student sample. Personality and Individual Differences, 46, 536-540.

Gonen-Yaacovi, G., de Souza, L.C., Levy, R., Urbanski, M., Josse, G., Volle, E., 2013.Rostral and caudal prefrontal contribution to creativity: a meta-analysis of functional imaging data. Frontiers in Human Neuroscience, 7, 465. 
Running head: Improvisation, divergent thinking, creativity and education

Gabora, L. (2005). Creative thought as a non-Darwinian evolutionary process. Journal of Creative Behavior, 39, 262-283.

Guilford, J. P. (1956). The structure of intellect. Psychological Bulletin, 53, 267-293.

Guilford, J.P., Christensen, P.R., Merrifield, P.R., \& Wilson, R.C. (1978). Alternate Uses: Manual of instructions and interpretations. Orange, CA: Sheridan Psychological Services.

H'Doubler, M.N. (1966). Dance: A Creative Art Experience. Wisconsin: University of Wisconsin Press.

Hambrick, D.Z., Oswald, F.L., Altmann, E.M., Meinz, E.J., Gobet, F., \& Campitelli, G. (2014). Deliberate practice: is that all it takes to become an expert? Intelligence, 45, 34-45.

Hanna, J. L. (1983). The mentality and matter of dance. Art Education, 36, 42-46.

Hassler, M. (1992). Creative musical behavior and sex hormones: musical talent and spatial ability in the two sexes. Psychoneuroendocrinology, 17, 55-70.

Henley, D. (2012). Cultural education in England. An independent review by Darren Henley for the Department for Culture, Media and Sport and the Department for Education, UK. London, UK: Department for Culture, Media and Sport.

Howard-Jones, P. A. (2002). A dual-state model of creative cognition for supporting strategies that foster creativity in the classroom. International Journal of Technology and Design Education, $12,215-226$.

Jaschke, A. C., Eggermont, L. H., Honing, H., \& Scherder, E. J. (2013). Music education and its effect on intellectual abilities in children: a systematic review. Reviews in the Neurosciences, 24, 665-675.

Jauk, E., Benedek, M., Neubauer, A.C. (2014). The road to creative achievement: a latent variable model of ability and personality predictors. European Journal of Personality, 28, 95-105. Jonides, J. (2008). Musical skill and Cognition. In C. Asbury \& C. Rich (Eds.) Learning, Arts, and the Brain. New York, NY: Dana Foundation. pp. 11-16.

Kaufman, J. C. \& Beghetto, R. (2009). Beyond big and little: The four c model of creativity. Review of General Psychology, 13, 1-12. 
Running head: Improvisation, divergent thinking, creativity and education

Karakelle, S. (2009). Enhancing fluent and flexible thinking through the creative drama process. Thinking Skills and Creativity, 4, 124-129.

Keinanen, M., Hetland, L., \& Winner, E. (2000). Teaching Cognitive Skill through Dance: Evidence for near but Not Far Transfer. Journal of Aesthetic Education, 34, 295-306.

Kim, J. (2002). The Effects of Creative Dance Instruction on Creative and Critical Thinking of Seventh Grade Female Students in Seoul, Korea. In R. J. Deasy (Ed.) Critical Links: Learning in the Arts and Student Academic and Social Development. Washington, DC: Arts Education Partnership.

Kim, K.H. (2005). Can only intelligent people be creative? Journal of Secondary Gifted Education, 16, 57-66.

Koutsoupidou, T., \& Hargreaves, D.J. (2009). An experimental study of the effects of improvisation on the development of children's creative thinking in music. Psychology of Music, 37, 251278.

Lewis, C. (2012). The Relationship Between Improvisation and Cognition. Unpublished PhD thesis, University of Hertfordshire.

Lewis, C., \& Lovatt, P. J. (2013). Breaking away from set patterns of thinking: Improvisation and divergent thinking. Thinking Skills and Creativity, 9, 46-58.

Liu, S., Chow, H.M., Xu, Y., Erkkinen, M.G., Swelt, K.E., Eagle, M.W., \& Braun, A.R. (2012). Neural correlates of lyrical improvisation: an fMRI study of freestyle rap. Nature: Scientific Reports, 2,834 .

Ma, H. (2009). The Effect Size of Variables Associated With Creativity: A Meta-Analysis. Creativity Research Journal, 21, 30-42.

Markey, P.M., Markey, C.N., Tinsley, B.J., \& Ericksen, A.J. (2002). A preliminary validation of preadolescents' self-reports using the Five-Factor model of personality. Journal of research in Personality, 36, 173-181.

McCrae, R. R., \& Costa, P. T. (1989). The structure of interpersonal traits: Wiggins's circumplex and the five-factor model. Journal of Personality and Social Psychology, 56, 586. 
Running head: Improvisation, divergent thinking, creativity and education

Mehta, R. \& Zhu, R. (2009). Red or Blue? Exploring the Effects of Color on Cognitive Task Performances. Science, 323, 1226-1229

Minton, S. (2002). Assessment of High School Students' Creative Thinking Skills: A Comparison of the Effects of Dance and Non-dance classes. In R. J. Deasy (Ed.) Critical Links: Learning in the Arts and Student Academic and Social Development. Washington, DC: Arts Education Partnership. Mosston, M., \& Ashworth, S. (2002). Teaching Physical Education. San Francisco, CA: CAB Cummings. National Endowment for the Arts (2014). Foundation for a New Arts Education Strategy: Education Leaders Institute Alumni Summit Report. Washington, DC: National Foundation for the Arts. NESTA (2012). Plan I: The Case for Innovation Led Growth. London, UK: NESTA.

Nijstad, B. A., De Dreu, C. K. W., Rietzschel, E. F. \& Baas, M. (2010). The dual pathway to creativity model: Creative ideation as a function of flexibility and persistence. European Review of Social Psychology, 21, 34-77.

Nooshin, L. (2003). Improvisation as' Other': Creativity, Knowledge and Power-The Case of Iranian Classical Music. Journal of the Royal Musical Association, 128, 242 - 296.

Partnership for $21^{\text {st }}$ Century Skills (2008). $21^{\text {st }}$ century skills, education \& competitiveness: $A$ resource and policy guide. Tucson, AZ: Partnership for $21^{\text {st }}$ Century Skills.

Plucker, J. A. (1998). Beware of simple conclusions: The case for content generality of creativity. Creativity Research Journal, 11, 179-182.

Plucker, J.A., (1999). Is the proof in the pudding? Reanalyses of Torrance's (1958 to present) longitudinal data. Creativity Research Journal, 12, 103-114.

Plucker, J. A., Beghetto, R. A., \& Dow, G. T. (2004). Why isn't creativity more important to educational psychologists? Potentials, pitfalls, and future directions in creativity research. Educational Psychology, 39, 83-96.

Plucker, J. A., Qian, M., \& Wang, S. (2011). Is Originality in the Eye of the Beholder? Comparison of Scoring Techniques in the Assessment of Divergent Thinking. Journal of Creative Behavior, $45,1-22$. 
President's Committee on the Arts and Humanties (2011). Reinvesting in Arts Education: Winning America's Future Through Creative Schools. Washington, DC: President's Committee on the Arts and Humanities.

Pressing, J. (1987). Improvisation: Methods and models. In J. Slobada (Ed.), Generative Processes in Music. Oxford, UK: Oxford University Press.

Raven, J.C., Raven, J., \& Court, J.H. (1962). Coloured Progrssive Matrices. Oxford, UK: Oxford Psychologists Press.

Ritter, S. M., Damian, R. I., Simonton, D. K., van Baaren, R. B., Strick, M., Derks, J., et al. (2012). Diversifying experiences enhance cognitive flexibility. Journal of Experimental Social Psychology, 48, 961-964.

Roberts, P. (2006). Nurturing Creativity in Young People. A report for Andrew Adonis Parliamentary Under-Secretary of State for Education and Skills, James Purnell Minister for Creative Industries and Tourism and David Lammy Minister for Culture.

Robinson, K. (1999). All Our Futures: Creativity, Culture and Education. A report for the Rt. Hon David Blunkett MP Secretary of State for Education and Employment and for the Rt. Hon Chris Smith MP Secretary of State for Culture, Media and Sport.

Runco, M.A., \& Jaeger, G.J. (2012). The Standard Definition of Creativity. Creativity Research Journal, 24, 92-96.

Sawyer, R. K. (2008). Learning music from collaboration. International Journal of Educational Research, 47, 50-59.

Schellenberg, E. G. (2001). Music and nonmusical abilities. Annuals of the New York Academy of Sciences, 930, 355-371.

Schmidt, T., Goforth, E., \& DrewSource, K. (1975). Creative Dramatics and Creativity: An Experimental Study. Educational Theatre Journal, 27, 111-114.

Schwarz, N., \& Clore, G. L. (2003). Mood as information: 20 years later. Psychological Inquiry, 14, 296-303. 
Running head: Improvisation, divergent thinking, creativity and education

Scott, G., Leritz, L. E., \& Mumford, M. D. (2004). The effectiveness of creativity training: A quantitative review. Creativity Research Journal, 16, 361-388.

Sharp, C. \& Le Métais, J. (2000). The Arts, Creativity and Cultural Education: An International Perspective (International Review of Curriculum and Assessment Frameworks). London, UK: Demos.

Silvia, P. J. (2008). Another look at creativity and intelligence: Exploring higher-order models and probable confounds. Personality and Individual Differences, 44, 1012-1021.

Silvia, P. J., Winterstein, B. P., Willse, J. T., Barona, C. M., Cram, J. T., Hess, K. I., \& Richard, C. A. (2008). Assessing creativity with divergent thinking tasks: Exploring the reliability and validity of new subjective scoring methods. Psychology of Aesthetics, Creativity, and the Arts, 2, 68-85.

Simon, H.A., \& Chase, W.G. (1973). Skill in chess. American Scientist, 61, 394-403.

Simonton, D. K. (1999). Creativity as blind variation and selective retention: Is the creative process Darwinian? Psychological Inquiry, 10, 309-328.

Smithrim, K., \& Upitis, R. (2005). Learning through the arts: Lessons of engagement. Canadian Journal of Education, 28, 109-127.

Sowden, P. T. \& Dawson, L. (2011). Creative feelings: The effect of mood on creative ideation and evaluation. ACM: Creativity and Cognition, 2011, 393-394.

Sowden, P. T., Pringle, A., \& Gabora, L. (2015). The shifting sands of creative thinking: Connections to dual-process theory. Thinking \& Reasoning, 21, 40-60.

Steidle, A., \& Werth, L. (2013). Freedom from constraints: darkness and dim illumination promote creativity. Journal of Environmental Psychology, 35, 67-80.

Torrance, E. P. (1974). Torrance Tests of creative thinking. Directions manual and scoring guide, verbal test booklet B. Scholastic Testing Service. 
Running head: Improvisation, divergent thinking, creativity and education

van Breukelen, G.J.P. (2006). ANCOVA versus change from baseline had more power in randomized studies and more bias in nonrandomized studies. Journal of Clinical Epidemiology, 59, 920925.

van de Kamp, M.-T., Admiraal, W., van Drie, J. \& Rijlaarsdam, G. (2015). Enhancing divergent thinking in visual arts education: Effects of explicit instruction of meta-cognition. British Journal of Educational Psychology, 85, 47-58.

Vaughn, K. (2000). Music and mathematics: Modest support for the oft-claimed relationship. Journal of Aesthetic Education, 34, 149-166.

Vaughn, K., \& Winner, E. (2000). SAT scores of students who study the arts: What we can and cannot conclude about the association. Journal of Aesthetic Education, 34, 77-89.

Vohs, K. D., Redden, J. P., \& Rahinal, R. (2013). Physical order produces healthy choices, generosity, and conventionality, whereas disorder produces creativity. Psychological Science, 24, 18601867.

Wallach, M.A., \& Kogan, N. (1965). Modes of thinking in young children: a study of the creativityintelligence distinction. New York, NY: Holt, Rinehart \& Winston.

Warwick Commission (2015). Enriching Britain: Culture, Creativity and Growth. Coventry, UK: Warwick University.

Winner, E., \& Cooper, M. (2000). Mute those claims: No evidence (yet) for a causal link between arts study and academic achievement. Journal of Aesthetic Education, 34, 11-75.

Winner, E., \& Vincent-Lancrin, S. (2013). The impact of arts education: Evidence and agenda for future research. In E. Liebau, E. Wagner, and \& M. Wyman (Ed's) International Yearbook for Research in Arts Education Vol. 1. Münster, Germany: Waxman. pp. 71-78.

Zenasni, F., \& Lubart, T. I. (2009). Perception of emotion, alexithymia and creative potential. Personality and Individual Differences, 46, 353-358. 
Running head: Improvisation, divergent thinking, creativity and education

Table 1

Mean pleasure (1 is high, 9 is low) and arousal (1 is high, 9 is low) scores for each condition after dancing

\begin{tabular}{lll}
\hline Condition & $M$ pleasure $[95 \% \mathrm{Cl}]$ & $M$ arousal $[95 \% \mathrm{Cl}]$ \\
\hline Control & $1.69[.76,2.62]$ & $1.27[-.04,2.58]$ \\
Improvisation & $1.71[.82,2.61]$ & $1.43[.17,2.69]$ \\
\hline
\end{tabular}

